\title{
Knot the Usual Suspects: Finding the Diagrammatic Representations of Physical Knots
}

\author{
Silas Rickards, Teertho Bhattacharya, Grace Cheng, Joshua Valan, and \\ Zachary Webb \\ University of Florida
}

Faculty Mentor: Anne Donnelly, Center for Undergraduate Research

\begin{abstract}
In the last few hundred years, mathematicians have been attempting to describe the topological and algebraic properties of mathematical knots. Regarding the study of knots, there exists a disconnect between examining a knot's mathematical and physical definitions. This is due to the inherent difference in the topology of an open-ended physical knot and a closed mathematical knot. By closing the ends of a physical knot, this paper presents a method to break this discontinuity by establishing a clear relation between physical and mathematical knots. By joining the ends and applying Reidemeister moves, this paper will calculate the equivalent mathematical prime or composite knots for several commonly used physical knots. In the future, it will be possible to study the physical properties of these knots and their potential to expand the field of mathematical knot theory.
\end{abstract}

Keywords: knot, knots, knot theory, Reidemeister moves, physical knots

\section{Introduction}

In the subject of mathematics, the field of knot theory has become important in understanding key results in microbiology, chemistry, and physics, among others. Knots are just one-dimensional curves in three-dimensional space that do not intersect themselves anywhere. Knot Theory is the study of these curves, their topological properties, and the ways to manipulate them (Adams, 1994). Normally, mathematicians develop this theory within the realm of mathematics and apply it to real-world problems. The direction is usually constantly flowing outward from mathematics to physics and other sciences (Hirsch, 1996). However, what if it is possible to use principles of physics to aid in the creation of new results in knot theory? In this paper, a physical knot is defined as any knot that is commonly tied in the real world using rope or a similar material. Some examples include the bowline knot, the overhand knot, and the clove hitch. Physicists have studied many quantities of knots, such as the efficiency of retaining the 
rope tensile strength (Patil et. al., 2020), and this paper proposes that it may be possible to use some of these quantities to aid the field of mathematical knot theory.

To understand the importance and applications of knot theory, the origins and motives must be discussed. Physical knots have always been used for different tasks such as climbing or sailing, as well as in traditional cultures around the world. (Turner \& van de Griend, 1996) However, the first rigorous study of knots was done by Carl Friedrich Gauss when he developed the Gauss linking integral for computing the linking number of two knots (Ricca \& Nipoti, 2011). One of the first major applications of knot theory came from Lord Kelvin who suggested that atoms might consist of knotted vortex tubes of the ether, with each element corresponding to a different knot (Thomson, 1867). Furthermore, with Vaughan Jones' discovering his namesake Jones polynomial in 1984, interest from the mathematical community in knot theory continued to increase. Edward Witten discovered a new framework for the Jones polynomial, which made use of Feynman path integrals, and new applications of knot theory arose (Sossinsky, 2002). Finally, in 2012 Farhi et. al. found that the Alexander polynomial could be used to create "a concrete quantum money scheme based on superpositions of diagrams that encode oriented links with the same Alexander polynomial."

As stated in Colin C. Adam's "The Knot Book," knot theory also has many applications in chemistry. Potential applications to chemistry formed the initial motivation for developing knot theory and starting in the 1980s many researchers used the subject to realize important results in chemistry (Adams, 1994). According to Sumners, knot theory can be used to examine the various spatial isomers of molecules. Moreover, knot theory is also used to represent the flexibility of these long molecules (Sumners, 1987). Additionally, knot theory has many applications in biology, particularly in DNA topology (Adams, 1994). In Moore and Vazquez (2020), coherent band surgery, conversion of a knot into a two-component link, and noncoherent band surgery, which is similar to coherent band surgery except the orientations are not retained, is used in the study of low-dimensional topology, particularly in DNA topology.

There are plenty of examples of uses from knot theory pertaining to other sciences and to the physics of commonly used knots. In a study by Patil et. Al (2020), the researchers used optomechanical experiments to determine mechanical stability based on the changing color from mechanical deformations. They could predict stability in frequently encountered knots and tangles. Additionally, Santos et al. (2019) offered a good overview of where the current state of 
knot research lies. Hitch theory examines the frictional and mechanical forces involved in tying hitches. From a psychological approach, knots have been commonly used in puzzles and to test mathematical and spatial reasoning. Since knots are complicated to move and understand, the act of tying and untying them can become a good test for robotics algorithms.

Despite all the different types of knot research, there is a major research gap in terms of finding knot diagrams of physical knots. According to Patil et. Al (2020), “... there currently exists no comprehensive mathematical theory linking the topological and mechanical properties of knotted elastic structures." As far as the researchers can tell, there does not exist direct research studying the idea of joining the ends of a physical knot and determining the knot diagram of any physical knot. The goal of this paper is to transcribe many physical knots into a mathematical knot diagram to help lay the groundwork for future research into the relationship between physical knots and mathematical knot theory.

\section{Methodology}

This investigation aims to apply known mathematical knot theory to physical knots and explore the connections between virtual mathematical knots and physical knots. Two knots are ambient-isotopic if it is possible to continuously distort one knot into the other without breaking or passing through itself (Armstrong, 1979). Topologically speaking, all physical knots are ambient-isotopic to an untangled line of the same material. This can be proven by taking one end of a physical knot and pushing the end into itself in reverse to eliminate any crossings (Armstrong, 1979). In order to evaluate the physical knot in a mathematical context, the researchers take both free ends and stitch them together. This will create a mathematical knot. After manipulating this new mathematical knot using legal Reidemeister moves, the KLO (Knotlike Objects) software (Swenton, n.d.) aids in proof of knot equivalence between this knot and a known prime or composite mathematical knot.

\section{The Transformation of the Physical Knot}

This process of joining the two free ends of the physical knot assumes that the two ends are on the outside of the knot. In the case of physical knots where at least one end is within the enclosed knot, for example the bowline knot, joining the ends will create a new crossing. Since the ends are free to move, this crossing can be over or under a part of the knot; thereby creating 
two distinct knot diagrams per crossing. In this instance, the table will include both representations.

\section{Reidemeister Moves and Establishing Equivalence}

A Reidemeister move proof establishes equivalent prime knots for each physical knot diagram, as seen in Reidemeister's 1927's, “Elementare Begründung der Knotentheorie.”

The Reidemeister moves consist of 3 types: Type I (Figure 1)- the twisting or untwisting of a strand which will add or subtract a crossing; Type II (Figure 2)- poking the strand through another, which adds or subtracts 2 crossings; and Type III (Figure 3 )- moving a crossing over a separate strand, which does not change the number of crossings.

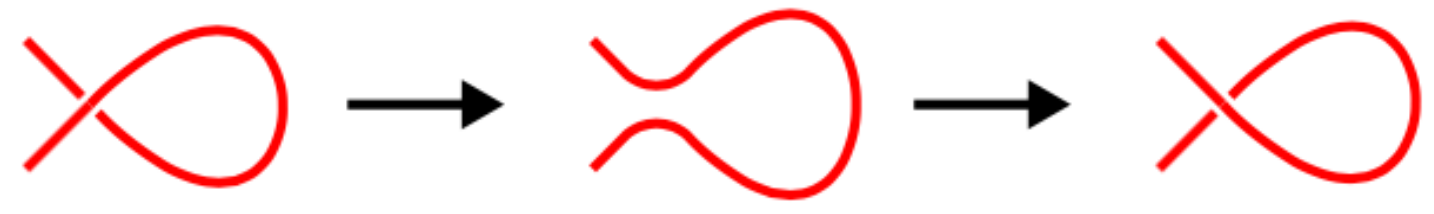

Figure 1. Reidemeister Type I (Reidemeister, 1932)

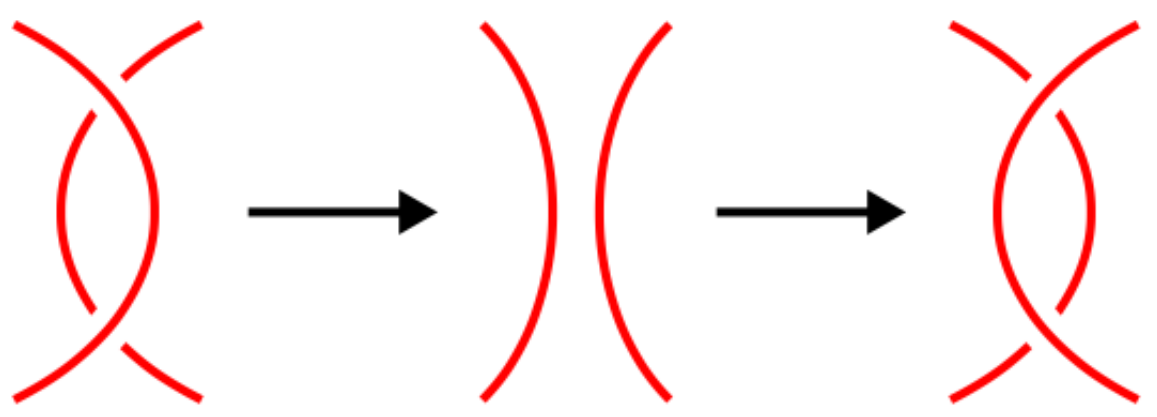

Figure 2. Reidemeister Type II (Reidemeister, 1932) 


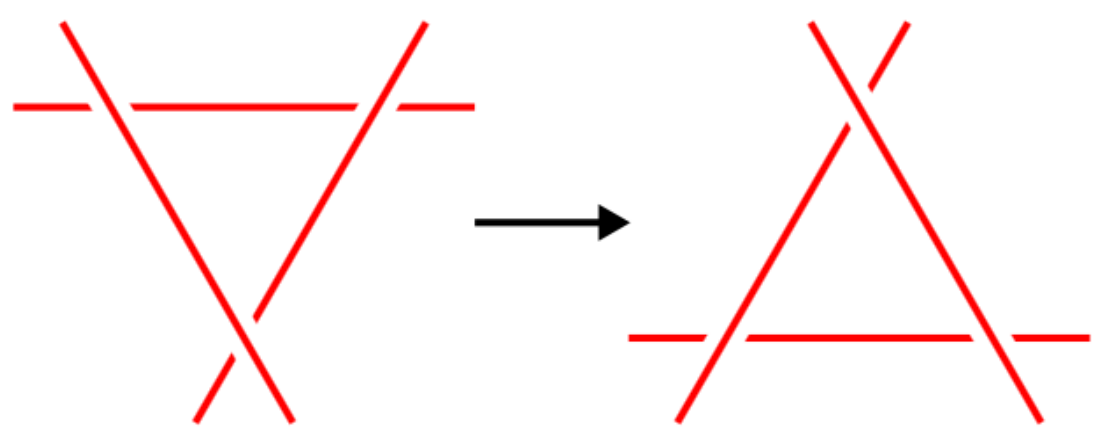

Figure 3. Reidemeister Type III (Reidemeister, 1932)

To construct a proof, the researchers will concurrently attempt to calculate several knot invariants and manipulate real rope to help guide the Reidemeister move sequence. By calculating the knot invariants, the list of possible prime and composite knots that may be equivalent to any given mathematical knot diagram becomes more feasible. The physical knot follows the structure of the recorded knot diagram. To emulate the form of a mathematical knot, free ends of physical knots must be joined. The alteration of the physical knot yields a mathematical model which can be manipulated in a firsthand manner. The rope is simply to be used as a guide to expedite the construction of the proof, where the sequence of Reidemeister moves leads to the conclusion of equivalence. Reidemeister moves performed firsthand in this physical manner will be legal, and therefore the proof extracted is valid.

Frank Swenton's KLO program aids in standardizing the process of simplifying knots. The program provides the simplification process using Reidemeister moves, and the program is the source of the virtual knot diagrams. This program cross-examines the physical manipulation mentioned above, where a physical rope emulates the mathematical knot in manipulation. Here, the program checks the proof in a virtual setting.

\section{Results}

Table 1 below details the findings of this investigation. The physical knots are organized in alphabetical order. The rightmost column contains the name and a graphic of the original, unaltered knot. The middle column contains the Reidemeister moves used to transform the knot, with the type of move indicated by Roman numerals I, II, and III. The altered regions of the knot are highlighted in brown. In the rightmost column is the equivalent prime or composite knot with the crossing number and index number underneath, respectively. 
Table 1. Conversion of Knots from Physical to Mathematical

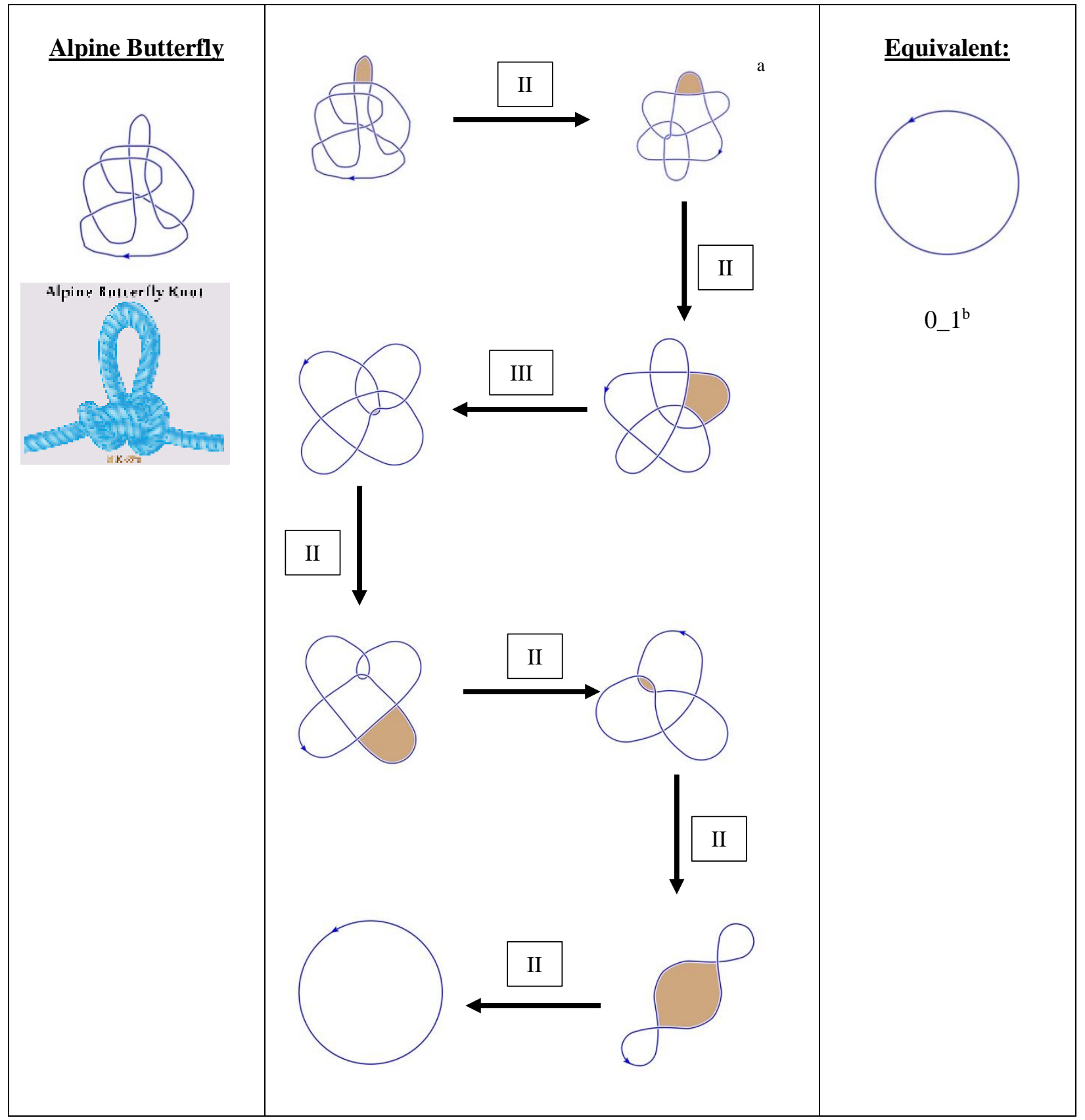




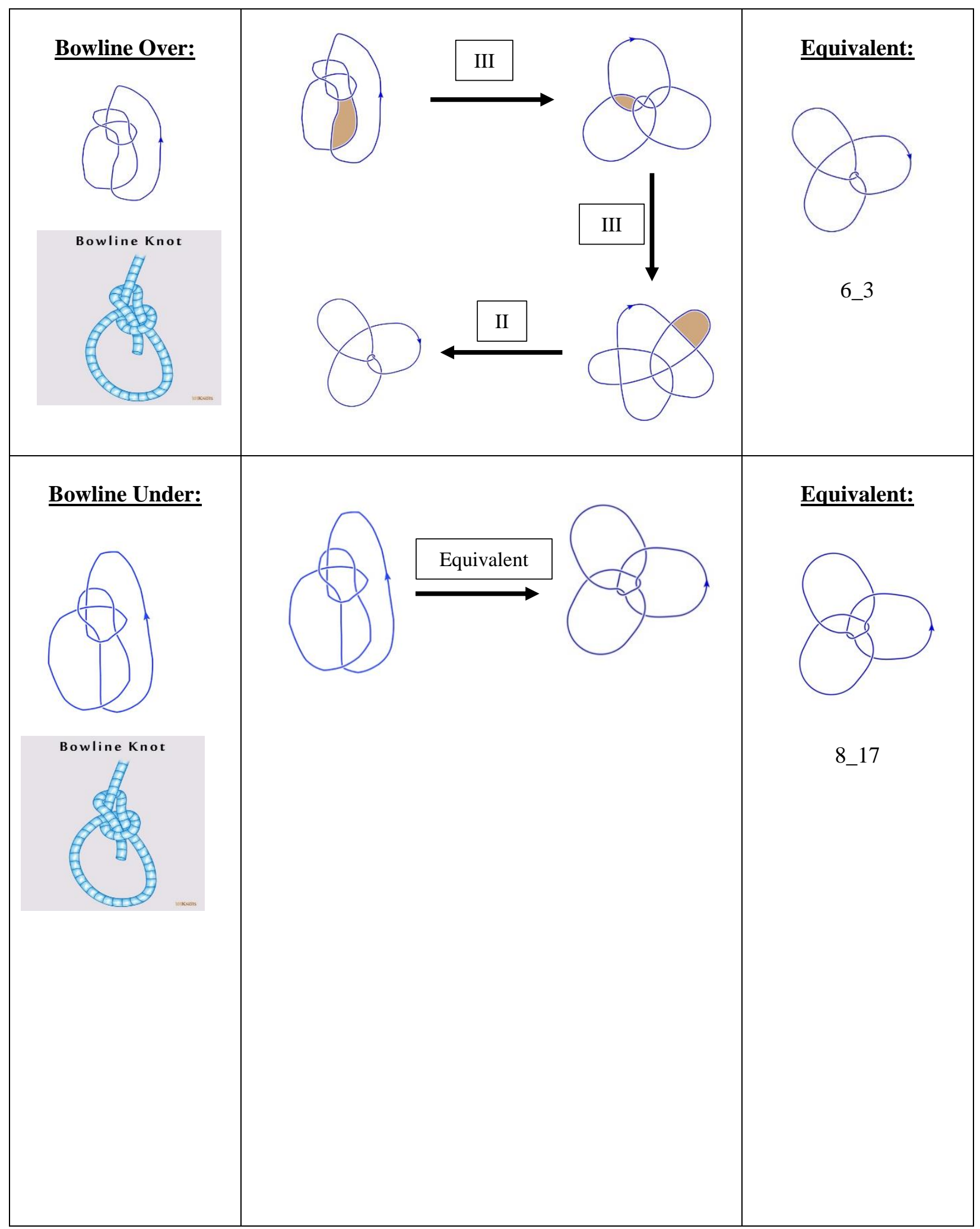




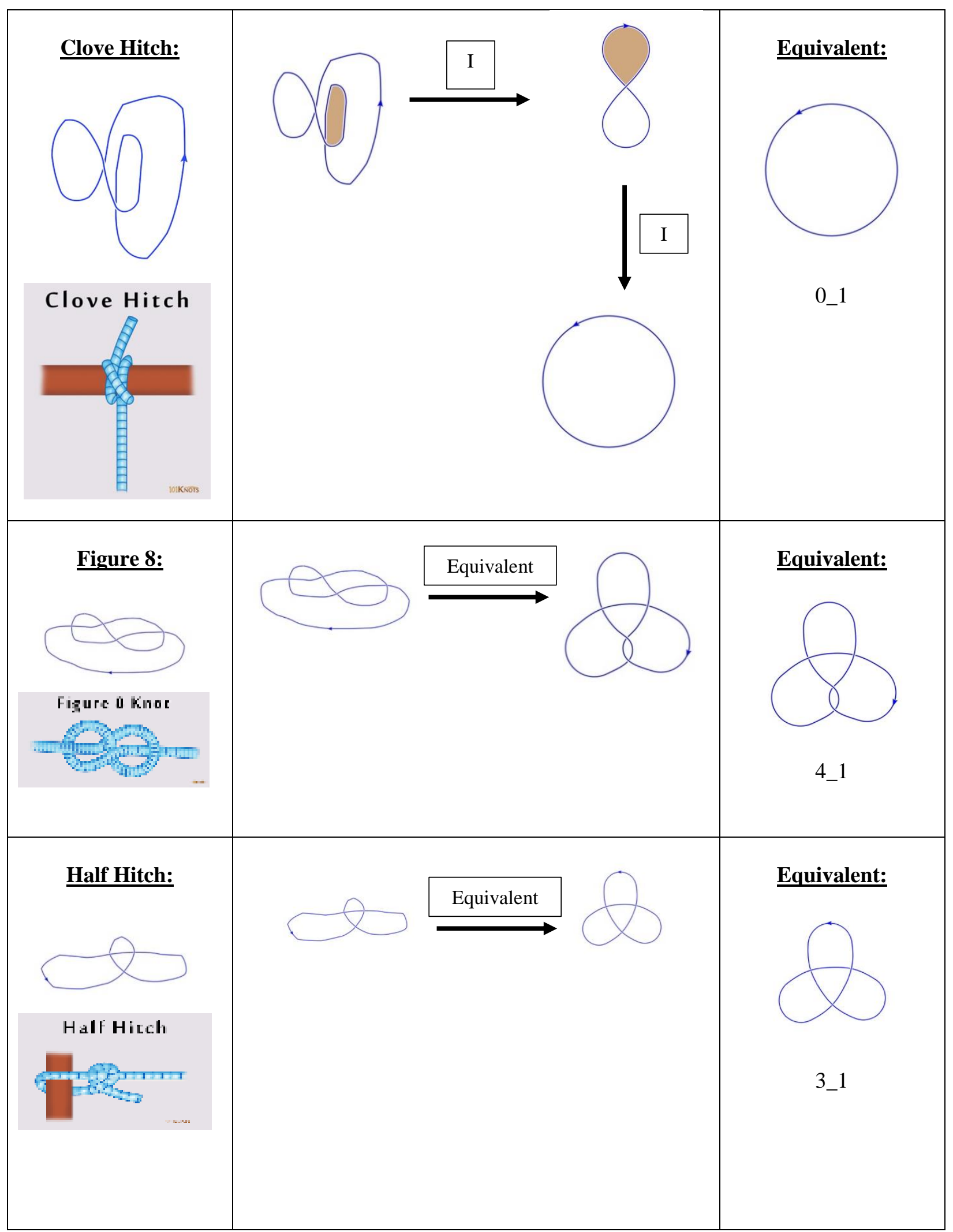


KNOT THE USUAL SUSPECTS: FINDING THE DIAGRAMMATIC REPRESENTATIONS OF

PHYSICAL KNOTS

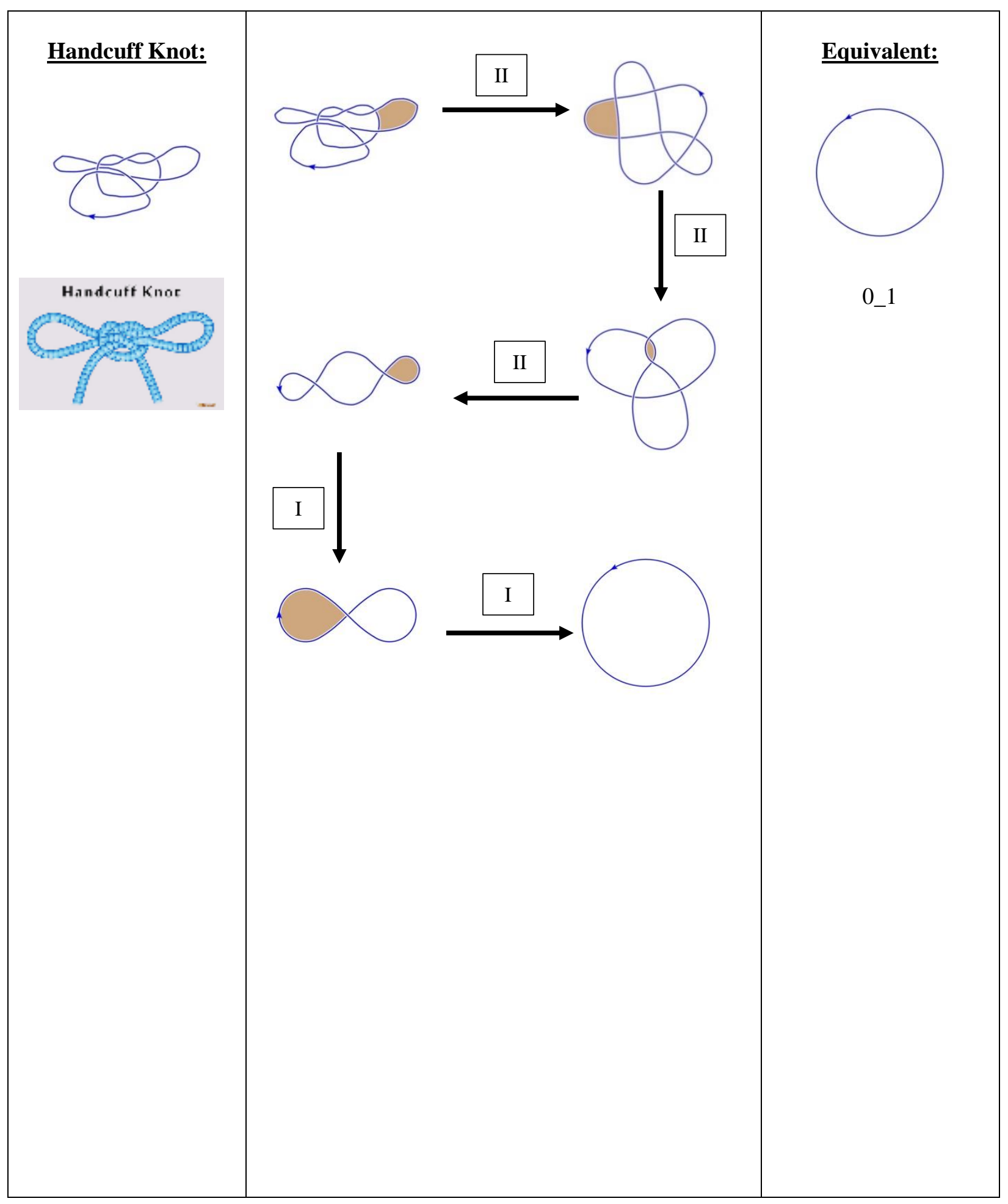




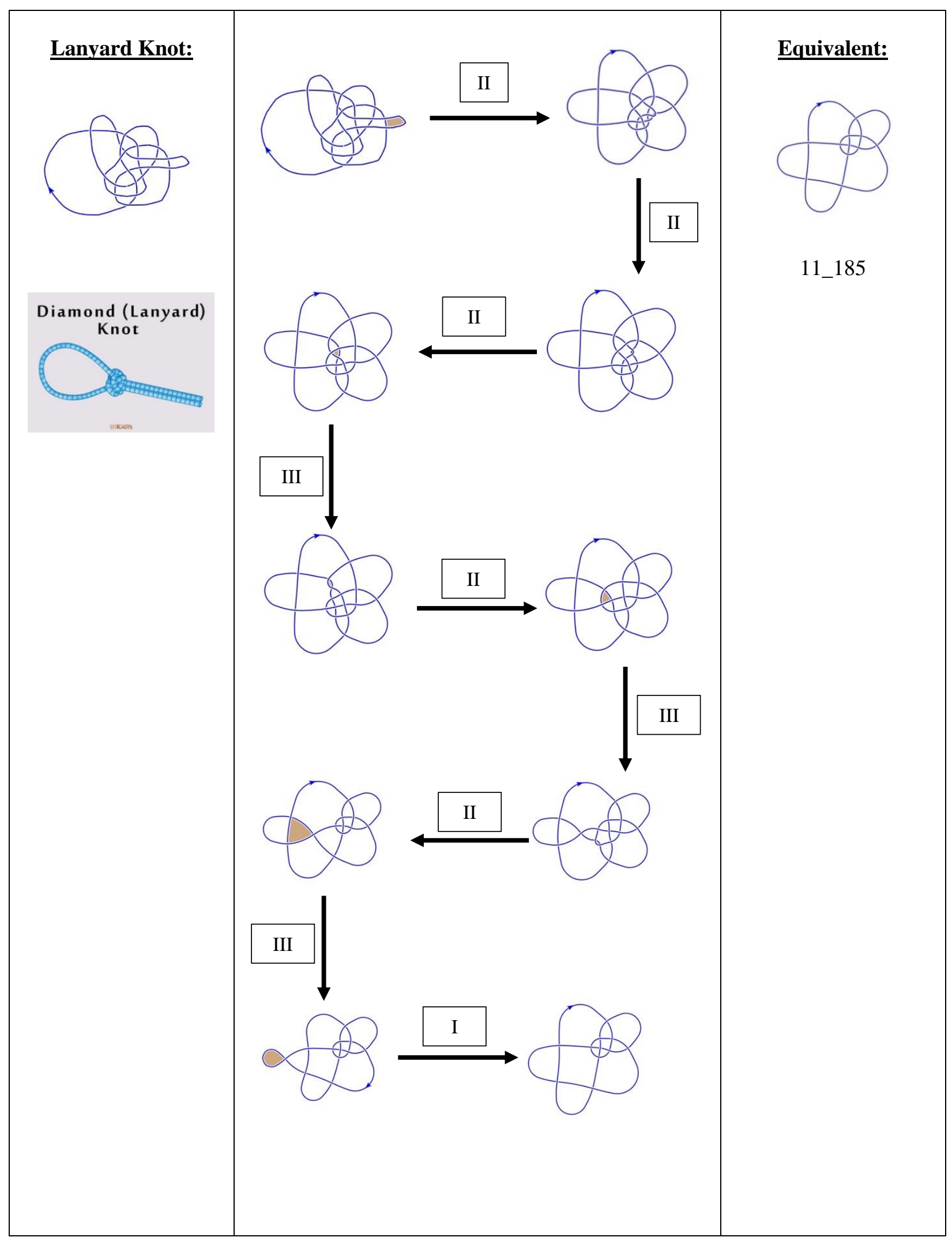




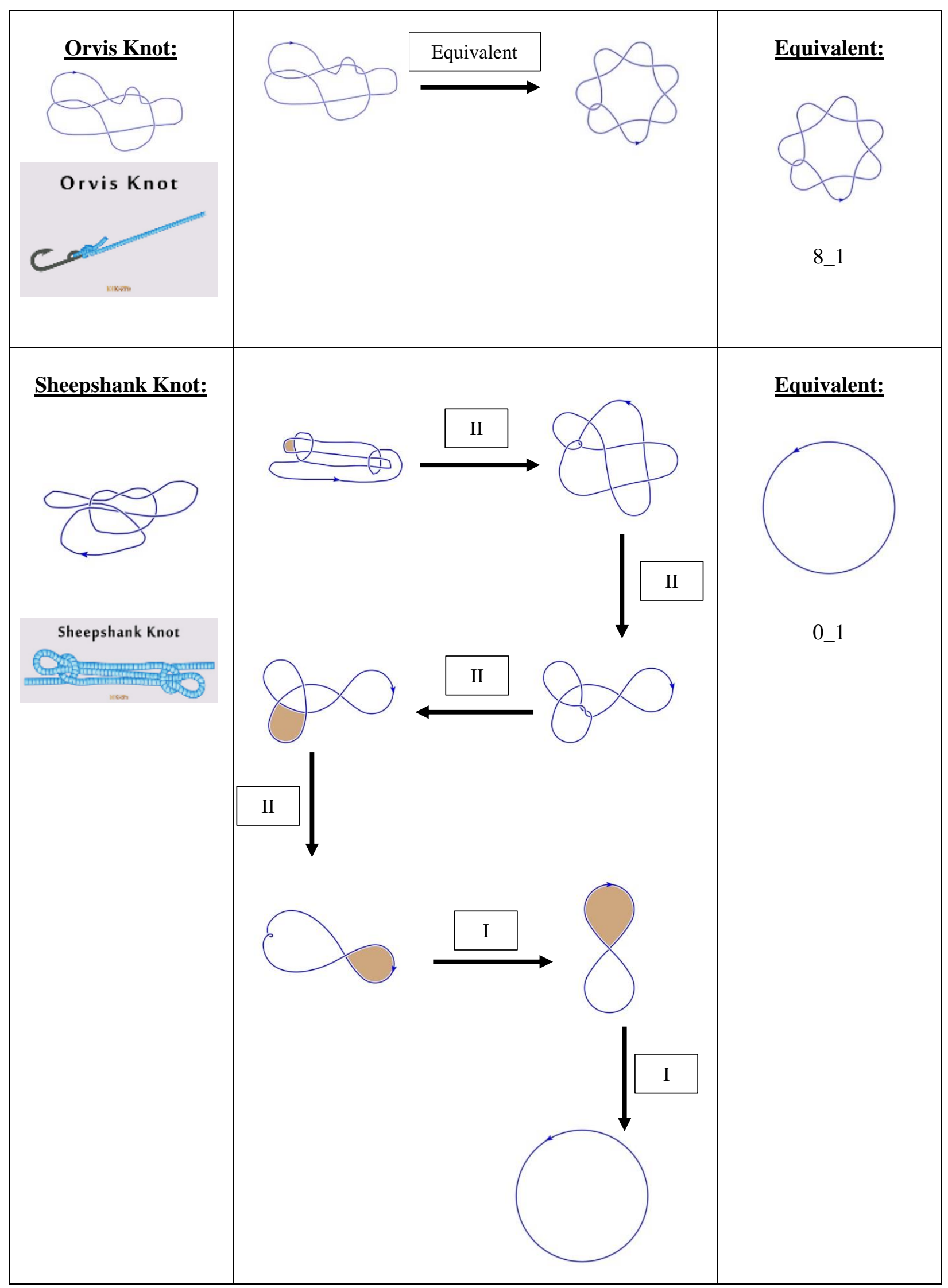




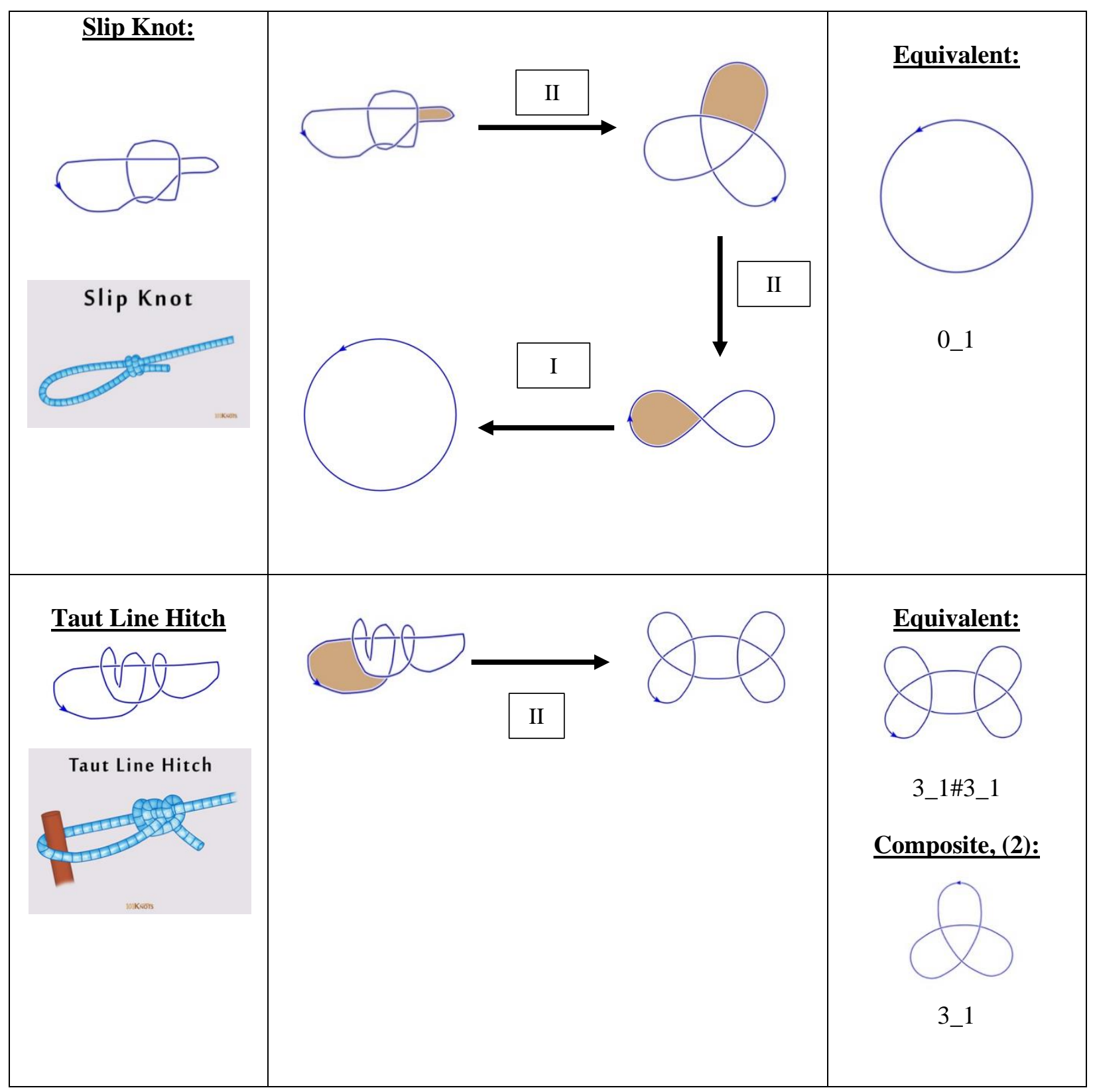

Table 1. Conversion of knots from physical to mathematical. This contains the knots' proofs of equivalence. aColumn 2: The brown highlight denotes the part of the knot that is going to be altered.

${ }^{b}$ Column 3: The first number indicated number of crossings in the knot, and the last number indicates the index number, or identifying number according to the Rolfsen knot table. 


\section{Discussion}

These results are the beginning of creating a link between mathematical and physical knots, which could further researcher's understanding of how real knots relate to each other. Since only 12 physical knots were studied in this paper, it would be best to take caution in any conclusions that are drawn based solely on this research. Even so, this paper is a foundation from which future research can be conducted. The fact that there exists at least two physical knots that, when their ends are joined, are ambient isotopic to the unknot is a promising lead.

Upon examination, some of the knots that are equivalent to the unknot, such as the sheepshank and slip knot, have similar physical characteristics. Specifically, the sheepshank knot shown is a particularly weak variation (Verrill, 2006) and the slip knot is normally a temporary measure, as pulling on one end will cause it to untie itself (Turner, 1996). The knots' tendency to untie themselves could have a connection to both the unknot mathematical model and the weaker nature of the knot in a physical application, considering factors such as friction, tension, and elasticity explored by Patil et. Al. (2020). The unstable nature of the crossings in the two knots could possibly be connected to the higher instance of Reidemeister move Type II in the proof, due to the poking of a strand through another resembling a slipping motion. Perhaps the similar utility of these knots is shown in their mathematical equivalent, though this needs further research.

However, there still exists knots which are equivalent to the unknot but have high strength and stability. Three knots in this investigation fit this description - the clove hitch, Alpine butterfly knot, and handcuff knot. Considering the uses of the knots as detailed by Verrill (2006), it is apparent that there are other objects which intersect loops in the knot, such as a carabiner in the Alpine butterfly knot, hands which intersect the handcuff knot, and a bar which intersects the clove hitch. The intersecting object would secure part of the knot, acting as a barrier to one or more loops, and this may partially differentiate the strength of these three knots from the weaker sheepshank and slip knots.

Concerning the different types of Reidemeister moves, Type III is the move that appears least in this investigation, but it is present in proofs of knots that can be used to hold human weight (Verrill, 2006). The bowline and Alpine butterfly knot are two such cases. Although not confirmed, the integrity of knots could possibly be gleaned through examining the properties and implications of the Reidemeister moves. 
The knots which are not equivalent to the unknot constitute slightly over half of the investigation. One knot in particular, the bowline, is one of the most diverse knots in application and is relatively simple to tie but still very reliable (Verrill, 2006). Although it is not clear why this is the case, it would be useful to examine the characteristics of the equivalent mathematical knots in conjunction with physical properties like those investigated by Patil et. Al. (2020), including twist fluctuations and circulations, which may be evident in the geometry of equivalent mathematical knots in column three as well as the original knot configurations in column one of Table 1.

Small changes to a mathematical or physical knot will lead to a change in the equivalent knot as well. With further understanding of the relationship between these knots, these changes could be predicted and understood. Whether it be in understanding the physical properties of real knots or the properties of mathematical knots, the knowledge of how both relate to each other will assist research in both fields.

\section{Limitations and Delimitations}

While visual matching is a workable technique, it certainly is not the optimal way to do it. For more certain matching between physical and mathematical knots, calculations of the invariants of the physical knot's prime counterpart will greatly improve finding the matching knot. Unfortunately, time constraints made this option unfeasible. Calculating the invariants proved challenging, and with a short deadline, visual mapping was chosen to have example knots completed in time. In the future, invariant calculation should be the technique for matching physical and mathematical knots. Not only would this remove human error present in visual matching, but it would also make searching easier. Invariants of the existing prime knots can be entered into a searchable database, and then entering the calculated invariants will lead to a much faster result.

While the techniques outlined here work for most knots, there are some limitations to what knots can be converted. Certain knots, such as the bowline, have one of their ends terminating inside of the knot itself. These knots cannot have their ends simply connected to form a mathematical knot like other knots. The solution used was to create two mathematical equivalents, one where the end exited the knot by going over the rest of the knot, and one where the end goes under the rest of the knot. This solution is workable, but it leads to two different equivalent knots and therefore no true equivalent like with the other knots. Additionally, this 
method can only be used on simpler knots. Matching the knots is done by comparing to the Rolfsen knot table visually, which grows almost exponentially as the number of crossings increases. The number of prime knots with 11 or more crossings makes it infeasible to find the equivalent using these methods. While it is still possible, the time investment with this method leaves it in dire need of improvement. This restriction is only present with the visual matching techniques. While creating a database such as the one outlined in the previous paragraph would be a large initial time investment at 11 and greater crossings, once it is created the time cost for larger knots is removed entirely.

\section{Conclusion}

In summation, the motive behind our research project was to fill the gap between physical knots and mathematical knots. Through studying the many types of non-intersecting curves in 3D space, mathematicians and physicists have been able to make incredible breakthroughs in science. With the use of legal Reidemeister moves, we were able to find the prime mathematical knot of 12 different physical knots. With this contribution to the body of knowledge, if one our knots is ever seen in nature, then there is an easy and accessible proof to convert it into a mathematical knot. The door to an interdisciplinary approach to knot theory is now open, and the opportunities for knot theory have increased ten-fold.

\section{Acknowledgements}

This paper would not have been possible without Dr. Anne Donnelly, Dr. Jennifer Moses, Dr. Frank Swenton, and Matthew Po. They helped guide the research to a smooth conclusion during the troubling times. 


\section{References}

Adams, C. C. (1994). The knot book: an elementary introduction to the mathematical theory of knots [Bibliographies Non-fiction]. W.H. Freeman.

Armstrong, M. A. (1979). Basic Topology. Springer New York.

Farhi, E., Gosset, D., Hassidim, A., Lutomirski, A., \& Shor, P. (2012). Quantum money from knots. ArXiv, abs/1004.5127.

Hirsch, R. (1996). Is Mathematics a Pure Science? Science \& Society,60(1), 58-79. http://www.jstor.org/stable/40403533

Moore, A. H., \& Vazquez, M. (2020). A note on band surgery and the signature of a knot. Bulletin of the London Mathematical Society, 52(6), 1191-1208. https://doi.org/https://doi.org/10.1112/blms.12397

Patil, V. P., Sandt, J. D., Kolle, M., \& Dunkel, J. (2020). Topological mechanics of knots and tangles. Science, 367(6473), 71-75. https://doi.org/10.1126/science.aaz0135

Reidemeister, K. Elementare Begründung der Knotentheorie. Abh.Math.Semin.Univ.Hambg. 5, 24-32 (1927). https://doi.org/10.1007/BF02952507

Reidemeister, Kurt. (1932). Knotentheorie. Ergebnisse der Mathematik.

Ricca, R. L., \& Nipoti, B. (2011). Gauss' Linking Number Revisited. Journal of Knot Theory and Its Ramifications, 20(10), 1325-1343. https://doi.org/10.1142/s0218216511009261

Santos, P. E., Cabalar, P., \& Casati, R. (2019). The knowledge of knots: an interdisciplinary literature review. Spatial Cognition \& Computation, 19(4), 334.

Sosinskiæi, A. B., Weiss, G., \& Nelson, M. C. (2002). Knots: Mathematics with a Twist. Cambridge, MA: Harvard University.

Sumners, D. (1987). The Knot Theory of Molecules. Journal of Mathematical Chemistry, 1-14. https://doi.org/10.1007/BF01205335

Swenton, Frank. (n.d). KLO software, version 0.969 alpha. https://community.middlebury.edu/ mathanimations/klo/

Thomson, W. (1867). On vortex atoms. Proceedings of the Royal Society of Edinburgh, 6, 94-105.

Turner, J. C., \& van de Griend, P. (Eds.). (1996) History and Science of Knots. World Scientific https://doi.org/10.1142/2940

Verrill, A. H. (2006). Knots, Splices and Rope-Work: An Illustrated Handbook. Dover Publications. 